\title{
BMJ Open Early psychosocial intervention in Alzheimer's disease: cost utility evaluation alongside the Danish Alzheimer's Intervention Study (DAISY)
}

\author{
Rikke Søgaard, ${ }^{1}$ Jan Sørensen, ${ }^{1}$ Frans B Waldorff, ${ }^{2,3}$ Ane Eckermann, ${ }^{2}$ \\ Dorte V Buss, ${ }^{2}$ Kieu T T Phung, ${ }^{2}$ Gunhild Waldemar ${ }^{2}$
}

To cite: Søgaard R, Sørensen J, Waldorff FB, et al. Early psychosocial intervention in Alzheimer's disease: cost utility evaluation alongside the Danish Alzheimer's Intervention Study (DAISY). BMJ Open 2014;4:e004105. doi:10.1136/bmjopen-2013004105

- Prepublication history for this paper is available online. To view these files please visit the journal online (http://dx.doi.org/10.1136/ bmjopen-2013-004105).

Received 23 September 2013 Revised 27 November 2013 Accepted 29 November 2013

CrossMark

For numbered affiliations see end of article.

Correspondence to Mr Jan Sørensen; jas@cast.sdu.dk

\section{ABSTRACT}

Objective: To assess the cost utility of early psychosocial intervention for patients with Alzheimer's disease and their primary caregivers.

Design: Cost utility evaluation alongside a multicentre, randomised controlled trial with 3 years of follow-up.

Setting: Primary care and memory clinics in five Danish districts.

Participants: 330 community-dwelling patients and their primary caregivers.

Intervention: Psychosocial counselling and support lasting 8-12 months after diagnosis and follow-up at $3,6,12$ and 36 months in the intervention group or follow-up only in the control group.

Main outcome measures: The primary outcome measure was the cost of additional quality-adjusted life years (QALYS). Costs were measured from a societal perspective, including the costs of healthcare, social care, informal care and production loss. QALYs were estimated separately for the patient and the caregiver before aggregation for the main analysis.

Results: None of the observed cost and QALY measures were significantly different between the intervention and control groups, although a tendency was noted for psychosocial care leading to cost increases with informal care that was not outweighed by the tendency for cost savings with formal care. The probability of psychosocial intervention being costeffective did not exceed $36 \%$ for any threshold value. The alternative scenario analysis showed that the probability of cost-effectiveness increased over the range of threshold values used if the cost perspective was restricted to formal healthcare.

Conclusions: A multifaceted, psychosocial intervention programme was found unlikely to be costeffective from a societal perspective. The recommendation for practice in settings that are similar to the Danish setting is to provide follow-up with referral to available local support programmes when needed, and to restrict large multifaceted intervention programmes to patients and caregivers with special needs until further evidence for cost-effectiveness emerges.

\section{Strengths and limitations of this study}

- Cost-utility analysis of a psychosocial intervention for individuals with newly diagnosed dementia and their relative based on a randomized controlled trial with 36 months of follow-up.

- Relatively high response rates combined with detailed register data from national registries provide comprehensive cost and utility data.

- The health utility of the patients was proxy rated, which has been shown to potentially imply an underestimation.

- The validity of the measurement of informal care warrants some consideration.

Trial registration: The study was registered in the Clinical Trial Database as ISRCTN74848736.

\section{INTRODUCTION}

According to the 2010 World Alzheimer's Report, more than 36 million people are living with dementia, and the expectations are that this number will have tripled by $2050 .{ }^{1}$ The associated economic impact is estimated to approach $€ 500$ billion/year, and to increase in proportion to the number of people living with dementia.

Alzheimer's disease (AD) accounts for approximately half of all cases of dementia. ${ }^{2}$ It is a progressive and irreversible disease, and even in the early phase, there is a substantial need for formal as well as informal care. A recent literature review demonstrated that the annual cost of care for mild AD ranged from approximately $€ 4000$ to approximately $€ 40000 .{ }^{3}$ Although the composition of these costs varied substantially 
across studies, it is clear that the cost burden of this disease had impacts far beyond the healthcare sector. Medical costs were found to constitute between $10 \%$ and $56 \%$ of the total costs, whereas non-medical costs constituted between $6 \%$ and $51 \%$ and the costs of informal care constituted between $8 \%$ and $81 \%$.

Until recently, the literature on effective management strategies for mild $\mathrm{AD}$ has focused on pharmacological treatment. However, the authors of a systematic literature review concluded that non-pharmacological strategies are useful, versatile and potentially cost-effective approaches to improve outcomes. ${ }^{4}$ One such approach was developed as part of the Danish Alzheimer's Intervention Study (DAISY) in 2004. Practitioners in the fields of geriatrics, psychiatry, neurology, psychology, care science, family medicine and law were involved in developing a psychosocial, supportive intervention to be tested in a randomised controlled trial. ${ }^{5}$ Clinical evaluation after 1 year of follow-up demonstrated no overall effect of the intervention, although it was concluded that longer term follow-up was needed. ${ }^{6}$ However, in a qualitative evaluation of the study, patients and caregivers indicated that they found the intervention stimulating and rewarding and that it helped them to find new ways to cope with the challenges of the disease. ${ }^{7}$ Now, after 3 years of follow-up, a long-term evaluation is reported in the current issue of this journal and concludes that there is no difference in clinical outcomes. ${ }^{8}$

The cost-effectiveness of non-pharmacological management of $\mathrm{AD}$ obviously relies on the clinical efficacy and on the cost consequences. Even if non-pharmacological management strategies fail to demonstrate the clinical benefits, there could be a rationale for adopting them if they address the escalating costs of $\mathrm{AD}$. In particular, studies in the North American setting have shown that caregiver support could delay nursing home placement, ${ }^{910}$ although other studies have not confirmed this finding. ${ }^{11} 12$

The European literature on the cost-effectiveness of non-pharmacological therapies for the treatment of mild $\mathrm{AD}$ appears to be very limited. The objective of this study was to assess the long-term cost utility of an early and intensive multifaceted, psychosocial intervention with follow-up support versus follow-up support alone for patients with $\mathrm{AD}$ and their primary caregivers from a societal perspective.

\section{METHODS}

\section{Study design and intervention}

The study was conducted alongside the randomised controlled multicentre trial of DAISY, which has been previously described in detail. ${ }^{5}$ Briefly, the trial included 330 dyads of patients and their primary caregivers, who were randomised to an intensive, multicomponent, semitailored psychosocial intervention programme with counselling, education and support (psychosocial care, 163 dyads) or to structured and systematic follow-up support (usual care, 167 dyads) and followed for 3 years. The inclusion criteria for the dyads were based on the characteristics of the patient and comprised age $\geq 50$ years, diagnosis of $\mathrm{AD}$, mixed $\mathrm{AD}$ diagnosis and vascular disease or dementia with Lewy bodies within the past 12 months, Mini-Mental State Examination score $\geq 20$ and possession of a primary caregiver who was willing to participate in the study. Institutionalised patients and patients with severe comorbidity were excluded. The psychosocial intervention was composed of individual and group-based counselling sessions using a constructivist approach, telephone counselling to the patient or the caregiver, a two-course series of five sessions each that targeted patients and caregivers individually, hand-outs with written information and the assignment of a contact person for each dyad for ad hoc monitoring and follow-up. The dyads in the control group as well as in the intervention group received follow-up visits at 3, 6, 12 and 36 months after randomisation. This means that both groups, in addition to usual care and the psychosocial intervention for those randomised to intervention, also received a follow-up intervention. At the assessments at 6 and 12 months, the raters were instructed to accommodate the patient's and the caregiver's typical frustration and uncertainty associated with a recent diagnosis by providing overall information and guidance, and they could facilitate contact with the relevant local support programmes.

\section{Costs}

Measurement of resource use was conducted from a societal perspective and was valued using opportunity costs to estimate the long-term average costs of providing the intervention on a routine basis. All costs were valued according to the price year 2008 and converted into EURO. The following is a summary of the methodology for establishing the cost parameter, which has been detailed in a separate report. ${ }^{13}$

The intervention cost was estimated from a microcosting procedure based on case reports with detailed time registrations, which were valued using loaded wage tariffs for health professionals. The microcosting procedure has also been reported in detail elsewhere. ${ }^{14}$

The costing of healthcare was based on national registers for service utilisation in the primary and secondary healthcare sectors. Tariffs of national agreements between Local Government Denmark and the respective professional associations (primary care) and tariffs of the Diagnostic-related-grouping (DRG) case-mix system (secondary care) were used for valuation. ${ }^{15}$

Events and duration of institutionalisation (in days) were extracted from national registries and validated by telephone interviews with relatives immediately after the end of the trial (eg, if the registers indicated that the patient had moved during the course of the trial). The item cost for a day at a nursing home was adapted from a large Swedish costing study that estimated the annual cost at 2005-SEK 472579 (2008-EUR 84 225). ${ }^{16}$ 
The Resource Utilisation in Dementia questionnaire ${ }^{17}$ was administered to caregivers at baseline and at 6,12 and 36 months after randomisation to monitor informal care and associated production loss. The questionnaire generally asked the respondent how much time was spent on a specific activity on a typical day and also the monthly frequency of such typical days. Accordingly, the total amount of time per month was calculated by multiplying these two measures, whereas the total for the whole study period was estimated using a linear interpolation between observations. Informal care and production loss were valued using opportunity cost of the caregivers' time, which was estimated from national age-matched and gender-matched net and gross wages, respectively.

\section{Utility}

The EuroQoL 5-dimensions (EQ-5D) was administered to caregivers at baseline and at 6, 12 and 36 months of follow-up for reporting of caregivers' health-related quality of life and for proxy reporting of patients' health-related quality of life. ${ }^{18}$ The collected descriptive classifications were converted into health utilities using the Danish scoring algorithm. ${ }^{19}$

QALYs were estimated individually for the patient and the caregiver as the area under the health utility curve over time using linear interpolation between observations, or between the last observation and zero if an individual was censored because of death. Lifetime was measured in days based on register data from the national registry of death causes. ${ }^{20}$

\section{Missing data}

Missing data on the questionnaire-based cost categories (informal care and production loss) and on the EQ-5D-based health utility scores were replaced using multiple imputation, which is generally used to address data missing at random. ${ }^{21}$ This method allows for uncertainty about missing data by creating, and appropriately combining the results from each of a number of different sets of plausible imputed data. It should be noted that the remaining cost categories (healthcare and institutionalisation) were based on administrative national registers with full coverage and were thus not subject to imputation. Additionally, it should be noted that costs and health utility were inherently zero from the time of death (which was identified from administrative registers) and that imputation also did not apply to these circumstances. During the follow-up, 66 dyads were censored because of the death of the patient or the caregiver; 40 dyads were in the intervention group and 26 were in the control group. The censoring was caused by patient death in 51 cases ( 34 in the intervention group and 17 in the control group) and caregiver death in 15 cases ( 6 in the intervention group and 9 in the control group). These numbers were different from those reported in the clinical report where dyads were censored because of death only if the patient died. ${ }^{8}$
Because all values missing as a result of death were scored as zero, the combined response rates of all cost variables and all health utility variables (for both members of each dyad) were $70 \%$ in the intervention group and $74 \%$ in the control group.

Logistic regression was used to identify the baseline characteristics that varied systematically between the responders and non-responders, which were used in the imputation procedure along with the non-missing responses. The baseline characteristics that were used as covariates included the following: age and gender of the patient, age and gender of the caregiver, whether the caregiver was working and the geographical affiliation of the dyad (ie, the particular centre in this multicentre study). Imputations were produced using the chained equation approach ${ }^{22}$ because the non-response was of a non-monotonic character (eg, non-respondents at time point one could return and become responders at time point two). The distributions for the incomplete cost categories were skewed and had a semicontinuous distribution, and a large proportion of patients had zero cost. These variables were split into the following two variables before imputation: a binary variable indicating whether the cost was zero and a log-transformed continuous variable for the actual cost when it was non-zero. Values for the binary and continuous variables were imputed and then multiplied to provide an imputed cost. The health utility scores were imputed on their original scale. Logistic regression was used to impute the binary variable of resource use and truncated linear regression was used to ensure that the imputations of the continuous variables of the costs and health utilities were within plausible ranges (eg, health utility was bound between -0.6 and 1 ). Twenty imputations were generated. After imputation, the complete cost data were transformed back to the original scale prior to the main analysis.

\section{Cost utility evaluation}

The cost utility evaluation was based on the standard methodology where costs and QALYs accrued over the follow-up period for each dyad were first quantified as individual parameters and then transformed into net benefit in order to estimate the cost-effectiveness of the psychosocial approach over the usual practice. The net benefit was estimated by valuing each dyad's QALY gain, using a range of hypothetical threshold values for decision-makers' willingness to pay for a QALY (from $£ 0$ to $£ 100000$ ) and subtracting the observed cost for the dyad. Both costs and QALYs were discounted at an annual rate of $3 \%$ (which is the consensus-based standard rate used in evaluations of Danish healthcare programmes).

The statistical analysis of the individual parameters and of the net benefit was based on non-parametric bootstrapping (10000 replications) due to the skewed nature of the data. ${ }^{23}$ For the QALY parameter, an adjustment for health utility at baseline was made to control 
for an average difference between the groups that was a result of statistical variation and not the treatment allocation. ${ }^{24}$ The adjustment was made by including baseline utility in regression models of treatment allocation on costs and QALYs, respectively.

Cost-effectiveness acceptability curves were used to illustrate the probability of the intervention being costeffective for the range of threshold values for willingness to pay for a QALY. ${ }^{25}{ }^{26}$ An alternative scenario analysis was conducted to test the methodological uncertainty of the imputation procedure, the choice of the cost perspective and the adjustment for baseline skewness of the randomisation. The impacts of these alternative scenarios were also illustrated using cost-effectiveness acceptability curves. All analyses were conducted using Stata V.12.1 for Mac.

\section{RESULTS}

\section{Costs and QALY differences}

The provision of early psychosocial intervention for patients with $\mathrm{AD}$ and their primary caregivers was estimated to incur an additional average cost of $€ 3401$. This estimate masked bidirectional tendencies, because cost savings were observed for the healthcare sector and for nursing home placement, whereas higher costs were observed for informal care. However, none of these findings were statistically significant. Table 1 details the costs of intervention, including the estimates of the complete case analysis, which led to even higher estimates for informal care. The higher estimates can be partly explained by the working status of caregivers with incomplete data, as they were often full-time workers and accordingly could not provide as many informal care hours as caregivers outside the labour force.

In terms of QALY, no statistically significant differences were observed between the groups. On average, however, the patients and caregivers in the intervention group reported lower scores than their counterparts in the control group. The lower scores were, to some extent, the result of a significant difference $(p<0.05)$ that already existed at baseline, which was controlled for in the reported differences of table 2. After adjustment for this baseline difference, the overall impact of the psychosocial intervention was estimated at an average negative value of 0.06 . The results of the complete case analysis supported the direction of this finding, although the findings based on complete cases were more extreme and led to a statistically significant $(\mathrm{p}<0.05)$ negative value of 0.38 . Figure 1 details the health utility scores over time and the impact of multiple imputations on the average values.

\section{Cost-effectiveness}

Given that the intervention did not seem to generate QALY gains or cost savings, the potential for costeffectiveness was limited. The statistical uncertainty surrounding these parameters, however, means that costeffectiveness cannot be ruled out until the combined uncertainty has been examined. Figure 2 shows the probability of the intervention being cost-effective at a continuum of hypothetical threshold values for decisionmakers' willingness to pay for an additional QALY. The curves declined for increasing threshold values because of the typical observation that the intervention led to a negative QALY gain. The probability for costeffectiveness did not exceed $36 \%$ for the imputationbased analysis and 14\% for the complete case analysis over the range of threshold values.

\section{Alternative scenario analysis}

A final set of uncertainties related to the main finding involved methodological uncertainties. Figure 3 shows the impact of alternative scenarios on the costeffectiveness acceptability curves. The scenario most

Table 1 Costs of care for dyads randomised to psychosocial intervention or control during 3 years of follow-up $(€)$

\begin{tabular}{|c|c|c|c|c|c|}
\hline & \multicolumn{2}{|c|}{ Psychosocial intervention } & \multicolumn{2}{|c|}{ Control } & \multirow{2}{*}{$\begin{array}{l}\text { Difference* } \\
\text { Mean (SE) }\end{array}$} \\
\hline & $\overline{\mathbf{n}}$ & Mean (SE) & $\mathbf{n}$ & Mean (SE) & \\
\hline \multicolumn{6}{|l|}{ Complete case analysis } \\
\hline Intervention & 92 & $1088(29)$ & 103 & 0 & $1088(29)$ \\
\hline Healthcare services & 92 & 11472 (1249) & 103 & $14546(1521)$ & $-3074(1983)$ \\
\hline Nursing homes & 92 & 21217 (5329) & 103 & 20189 (4092) & 1029 (6708) \\
\hline Informal care & 92 & $63092(10701)$ & 103 & 46931 (7670) & $16161(13246)$ \\
\hline Production loss & 92 & $2823(1040)$ & 103 & $2679(886)$ & 144 (1362) \\
\hline Total cost & 92 & $99692(11528)$ & 103 & 84344 (8594) & $15348(14401)$ \\
\hline \multicolumn{6}{|c|}{ Multiple imputation-based analysis } \\
\hline Intervention & 163 & $1031(26)$ & 167 & 0 & $1031(26)$ \\
\hline Healthcare services & 163 & 13597 (1196) & 167 & 15069 (1562) & $-1472(1976)$ \\
\hline Nursing homes & 163 & 20412 (3713) & 167 & 24404 (3577) & $-3992(5131)$ \\
\hline Informal care & 163 & 46557 (6346) & 167 & 38510 (4909) & 8047 (8066) \\
\hline Production loss & 163 & $2545(631)$ & 167 & 2758 (605) & $-214(882)$ \\
\hline Total cost & 163 & 84142 (6992) & 167 & 80741 (6029) & 3401 (9216) \\
\hline
\end{tabular}


Table 2 Quality-adjusted life years (QALY) for dyads randomised to psychosocial intervention or control during 3 years of follow-up

\begin{tabular}{|c|c|c|c|c|c|}
\hline & \multicolumn{2}{|c|}{$\begin{array}{l}\text { Psychosocial } \\
\text { intervention }\end{array}$} & \multicolumn{2}{|c|}{ Control } & \multirow{2}{*}{$\begin{array}{l}\text { Difference† } \\
\text { Mean (SE) }\end{array}$} \\
\hline & $\mathrm{n}$ & Mean (SE) & $\bar{n}$ & Mean (SE) & \\
\hline \multicolumn{6}{|c|}{ Complete case analysis } \\
\hline Patient QALY & 101 & $1.43(0.07)$ & 113 & $1.67(0.05)$ & $-0.17(0.07)^{\star}$ \\
\hline Caregiver QALY & 101 & $2.04(0.07)$ & 113 & $2.24(0.06)$ & $-0.19(0.09)^{\star}$ \\
\hline Total QALY & 101 & $3.47(0.13)$ & 113 & $3.92(0.09)$ & $-0.38(0.14)^{\star}$ \\
\hline \multicolumn{6}{|c|}{ Multiple imputation-based analysis } \\
\hline Patient QALY & 163 & $1.34(0.05)$ & 167 & $1.48(0.05)$ & $-0.06(0.06)$ \\
\hline Caregiver QALY & 163 & $1.92(0.06)$ & 167 & $1.98(0.06)$ & $-0.03(0.08)$ \\
\hline Total QALY & 163 & $3.26(0.10)$ & 167 & $3.46(0.10)$ & $-0.09(0.13)$ \\
\hline
\end{tabular}

likely to affect the main finding was a restriction to a cost perspective for formal care (ie, health-care and nursing home placement). In this scenario, the maximum probability for cost-effectiveness reached $78 \%$ for a zero threshold. Cost savings drove this probability, and it should be noted that the probability decreased for any value put on a QALY.

\section{DISCUSSION}

This study assessed the cost-effectiveness of early psychosocial intervention in patients with $\mathrm{AD}$. The main finding was that a psychosocial intervention is unlikely to be cost-effective in a Danish setting because it did not generate additional QALYs and it led to higher average usage of informal care.

\section{Strengths and weaknesses of the study}

The cost utility evaluation was conducted alongside a well-characterised clinical trial ${ }^{5}$ that represented one of the first large-scale randomised controlled trials conducted in strict adherence to the CONSORT guideline. Furthermore, it is the first report on the long-term cost-utility of a psychosocial intervention. Despite the usual challenges associated with assessing the effects of social care aimed at community-dwelling individuals, relatively high response rates were obtained. The responses were supported with detailed register data from national registries to provide a comprehensive data set that had been thoroughly analysed and separately interpreted before it was synthesised in this paper. 5681314

Informal care appeared to play an important role in the analysis of cost-effectiveness. Although it did not drive the main finding alone, the validity of the measurement of informal care warrants some consideration. A validated and well-known instrument ${ }^{17}$ was used for the measurement of informal care; however, it can be difficult to obtain unbiased estimates for a number of reasons. First, caregivers may not consider their life together with the person with dementia as service provision. Second, it is not possible over long periods of follow-up to monitor status with a frequency that rules out recall and interpolation bias. Third, there is much controversy regarding the valuation of informal care. ${ }^{27}$ However, it should be noted that even a zero valuation of informal care would not have altered the main finding.

The measurement of QALYs is similarly challenged by the long period of follow-up and the fact that the patient and the caregiver were subject to measurement. The health utility of the patients was proxy rated, which has been shown to potentially imply an underestimation. ${ }^{28}$ However, in this study there was no significant effect of the DAISY intervention on any quality of life measure. Based on results in a previous report ${ }^{29}$ we found that proxy rated quality of life probably better reflects progression of clinical measures as compared to patient-rated measures, and that EQ-5D is a suitable alternative to disease-specific quality of life measures in research. Additionally, it was assumed that the average trend between the observation points was linear. Although this is a standard practice in economic evaluation, AD could be a special case because of the disease progress and/or the ability of the caregiver to cope being non-linear for the observation points chosen in the present design. Nonetheless, any bias in the reported QALYs would have to affect the groups differently to weaken the validity of the analytical findings.

Patients in the intervention group had higher mortality than those in the control group although this increased mortality was unlikely to be caused by the intervention, as the nature of the intervention programme did not subject the patients to any health risk. At baseline, the quality of life of the patients in the intervention group was rated as poorer than that of the control group, by the patients themselves and by their caregivers. There were small insignificant differences in socioeconomic and clinical variables which could be associated with the higher mortality rate in the intervention group. More patients in the intervention group lived alone (4\% difference), rented their house $(7 \%$ difference), had more comorbidities (4.4\% difference) and were diagnosed with mixed $\mathrm{AD}$ and vascular 


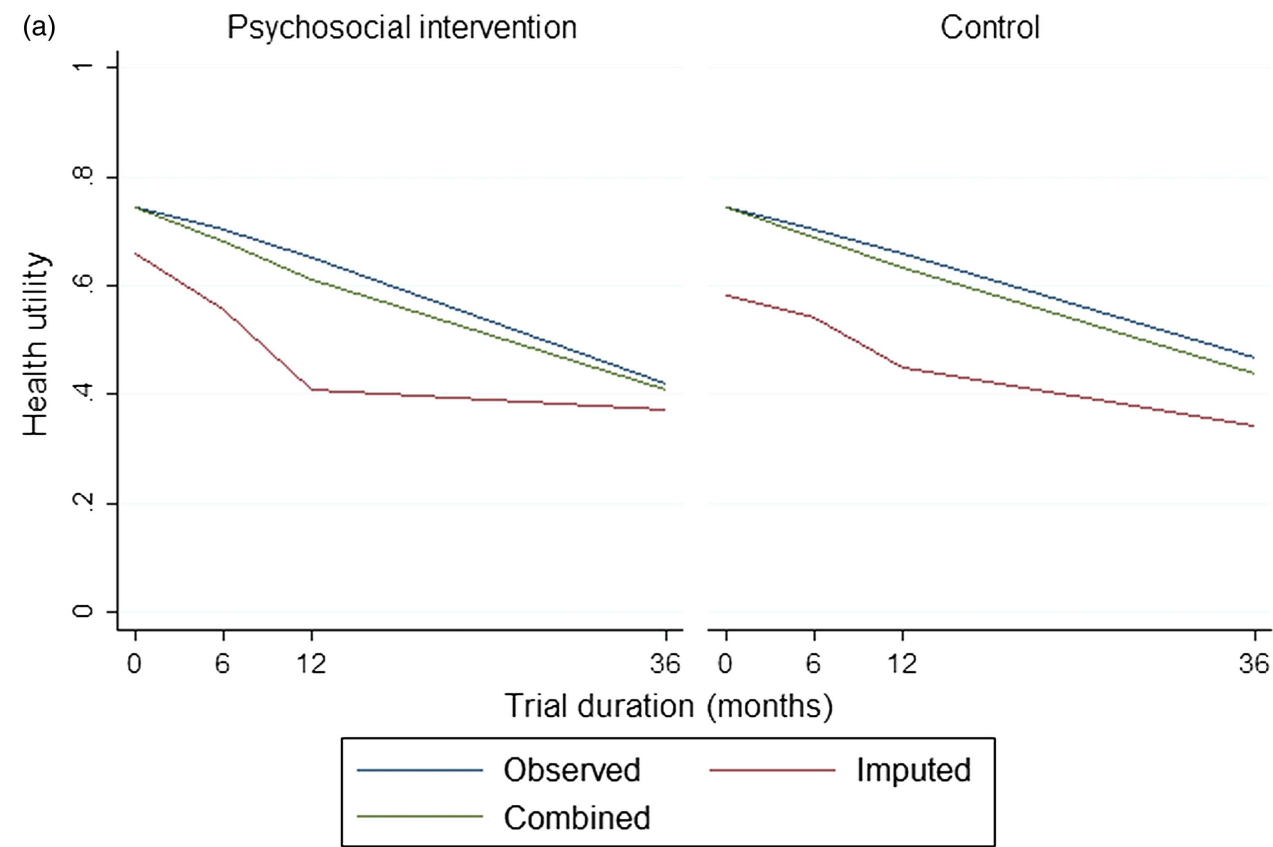

Graphs by ret

(b)

Psychosocial intervention

Control

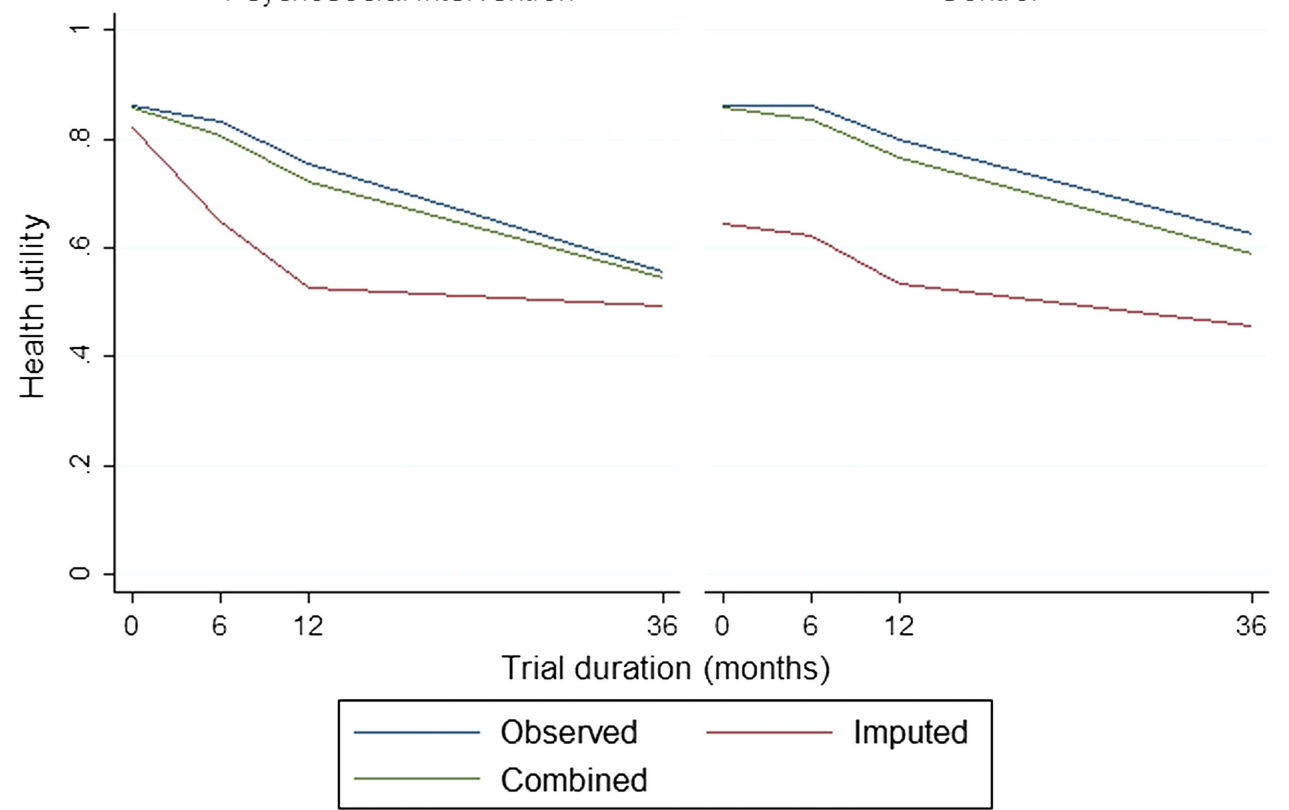

Graphs by rct

Figure 1 Observed health utility scores in the intervention and control groups: the panels show the values for $(A)$ patients and (B) caregivers.

dementia (4.2\% difference). Whether these differences could contribute to the higher mortality in the intervention group is uncertain.

The validity of the imputation procedure was based on the assumption that the data were missing at random, that is, that non-response was completely random when adjusted for observed covariates. A number of covariates appeared to explain the non-response and these covariates were associated with substantially lower health utility scores almost consistently over the entire follow-up period. This is shown in figure 1 where the influence of taking this appropriately into account is demonstrated to lower the average scores of both randomisation groups. The missing-at-random assumption was tested by a series of regressions of the parameters with missing values on the covariates used in the multiple imputation procedure and a dummy for whether a respondent had $\geq 1$ missing values. The fact that 'missingness' was not 


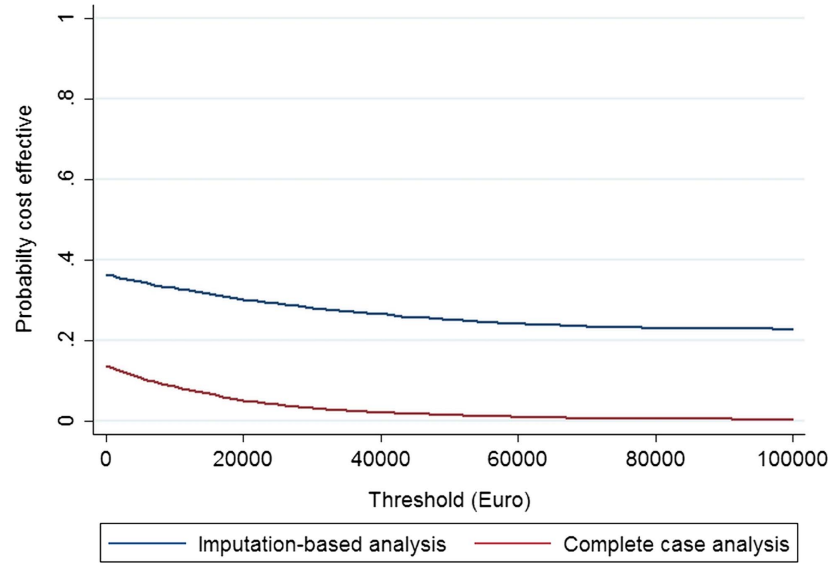

Figure 2 Cost-effectiveness acceptability curves for psychosocial intervention.

associated with the observed parameter values in any of these regressions gives some credibility that the 'missingness' is fully controlled for.

For ethical reasons the study design included a minimum of support for the control group in relation to the systematic assessment of their status at 6 and 12 months post randomisation. This is not a perfect reflection of the usual practice and could have led dyads in the control group to perform better than they would have without this minimum level of support.

\section{Comparison with other studies}

This study appears to be the first of its kind in assessing the long-term consequences of psychosocial intervention in mild AD. There have been, however, a few costeffectiveness evaluations on related therapies. A Dutch study investigated the cost-effectiveness of occupational therapy for patients with mild to moderate $\mathrm{AD}$ and for their primary caregivers. ${ }^{30}$ The study concluded that occupational therapy was cost-effective due to reducing the amount of informal care within the 3 months follow-up time. A recent UK study concluded that an intervention based on reminiscence groups was not cost-

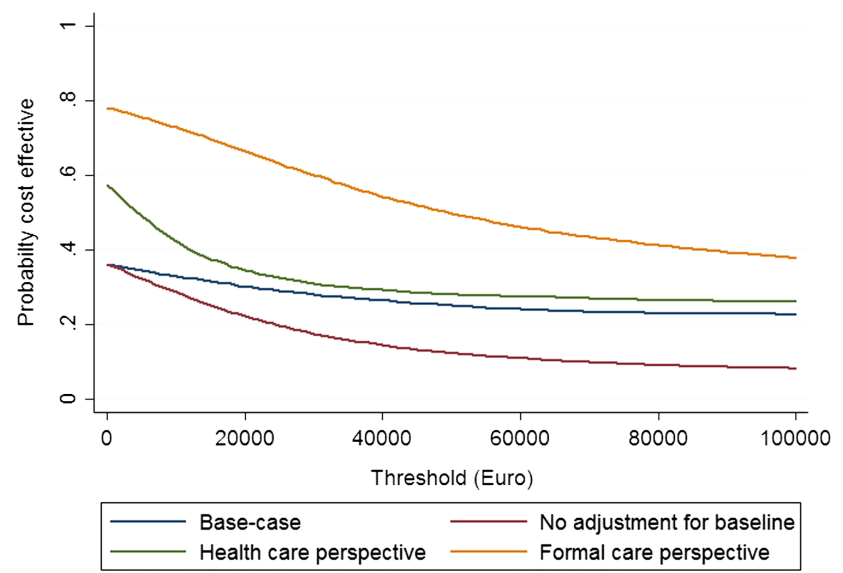

Figure 3 Cost-effectiveness acceptability curves for alternative analytical scenarios. effective, based on a cost perspective restricted to selected formal care. ${ }^{31}$ Finally, two Markov model-based studies have been reported. In a Finnish study on the cost-effectiveness of a family-support programme it was concluded that family support could be cost-effective because of overall cost savings. ${ }^{32}$ In a North American study the net benefit of three experimental management strategies in comparison with the usual care was assessed: pharmacological treatment, caregiver support and a combination of the two. ${ }^{33}$ Positive net benefits were identified for all of the experimental strategies and, in particular, the combined strategy provided a net benefit of up to $\$ 94000$ for a typical person with mild AD.

\section{Limitations and implications for practice}

In an assessment of external validity, it should be considered whether caregivers could have a higher or a lower need for intervention than in the present structural and cultural setting. The present setting is representative of a country with one of the world's largest public sectors where citizens have free access to a range of public services that could, to some extent, reduce the need for (extra) tailored support. In addition, Danish citizens are relatively well educated, and it is common for men and women to participate in the labour force. It is the authors' belief that the main findings are generalisable to other (North) European countries, especially those with a National Health Service system, whereas the descriptive costs cannot be expected to be generalisable across settings.

Given the negative finding of this study, which seems to be scientifically robust despite the challenges of conducting experimental research in the community setting, a recommendation for practice in settings that are similar to the Danish setting is to provide a follow-up with referral to available local support programmes when needed and to restrict large multifaceted intervention programmes to patients and caregivers with special needs until further evidence for cost-effectiveness emerges.

Author affiliations

${ }^{1}$ CAST - Centre for Applied Health Services Research, University of Southern Denmark, Odense, Denmark

${ }^{2}$ The Memory Disorders Research Group, Department of Neurology, Danish Dementia Research Centre, Copenhagen University Hospital, Copenhagen, Denmark

${ }^{3}$ Research Unit and Department of General Practice, Institute of Public Health, University of Copenhagen, Copenhagen, Denmark

Acknowledgements The DAISY study was supported by the National Board of Social Services at the Danish Ministry of Social Affairs, the Danish Ministry of Health and the Danish Health Foundation. The authors would like to thank the 128 volunteer raters and assistants from 68 municipalities and five counties, as well as the following project coordinators and physicians in the five participating centres for their contributions to patient recruitment, intervention and data collection: Ribe County. Esbjerg Hospital, Department of Psychiatry (Anna Marie Hansen, Johanne Christensen and Jørgen Jensen); Ringkoebing County. Herning Hospital, Department of Psychiatry (Marianne Refslund, Birgitte Aagaard and Palle Lund) and Holstebro Hospital, Department of Psychiatry (Inge Lund Petersen and Finn Andersen); Roskilde 
County. Roskilde Hospital, Department of Geriatrics (Dorte Dyre, Lisbeth Petersen, Birgitte Froelund, Lise Korbo and Ellen Holm) and Department of Neurology (Kurt Luedorfft); Vestsjaelland County. Korsoer Hospital, Department of Geriatrics (Mette Lassen and Lars Laugesen) and Dianalund Hospital, Department of Psychiatry (Thyge Jensen and Ole Bjoern Skausig); Copenhagen Capital area: Hvidovre Hospital, Department of Geriatrics (Lillian Moerk Joergensen), Amager Hospital, Department of Geriatrics (Suzanne Sanders), Bispebjerg Hospital, Department of Geriatrics (Claus Moe†), Frederiksberg Community Health Care Centre (Ingrid Lauridsen), Frederiksberg Hospital, Department of Psychiatry (Rene Klysner), Glostrup Hospital, Department of Neurology, (Jens Feilberg), Rigshospitalet, and Department of Neurology (Ane Eckermann, Eva Illemann, Susanne Rishoej, Dorthe V Buus, Lisbeth Villemoes Soerensen and Peter Johannsen).

Contributors All the authors except RS were involved in the conception and design of the DAISY trial. RS conducted the data analysis and drafted the manuscript. All the authors have read and approved the final manuscript. RS and JS are the guarantors

Funding The DAISY study was supported by the National Board of Social Services at the Danish Ministry of Social Affairs, the Danish Ministry of Health and the Danish Health Foundation. All the researchers were independent from the funders. The funders were not involved in the collection, analysis and interpretation of data; the writing of the report; or the decision to submit the article for publication.

Competing interests None.

Ethics approval The DAISY trial was conducted with strict adherence to the Helsinki Declaration. According to the Danish Act on Research Ethics, approval from the regional ethical committee was not required. However, we presented the protocol to the regional ethical committee for Copenhagen and Frederiksberg municipalities. The committee decided that no approval was needed (ID No (KF) 02-005/04). The Danish Data Protection Agency approved the research database (ID No 2003-41-3178). The study was registered in the Clinical Trial Database (http://www.controlled-trials.com/ISRCTN74848736). All patients and caregivers gave informed consent to participate in the trial and the extended 36 month follow-up.

Provenance and peer review Not commissioned; externally peer reviewed.

Data sharing statement No additional data are available.

Open Access This is an Open Access article distributed in accordance with the Creative Commons Attribution Non Commercial (CC BY-NC 3.0) license, which permits others to distribute, remix, adapt, build upon this work noncommercially, and license their derivative works on different terms, provided the original work is properly cited and the use is non-commercial. See: http:// creativecommons.org/licenses/by-nc/3.0/

\section{REFERENCES}

1. Alzheimer's Disease International. World Alzheimer Report 2010: The global economic impact of dementia. Alzheimer's Disease International, 2010.

2. Jalbert JJ, Daiello LA, Lapane KL. Dementia of the Alzheimer type. Epidemiol Rev 2008;30:15-34

3. Jonsson L, Wimo A. The cost of dementia in Europe: a review of the evidence, and methodological considerations. Pharmacoeconomics 2009;27:391-403.

4. Olazaran J, Reisberg B, Clare L, et al. Nonpharmacological therapies in Alzheimer's disease: a systematic review of efficacy. Dement Geriatr Cogn Disord 2010;30:161-78.

5. Waldemar G, Waldorff FB, Buss DV, et al. The Danish Alzheimer intervention study: rationale, study design and baseline characteristics of the cohort. Neuroepidemiology 2011;36:52-61.

6. Waldorff FB, Buss DV, Eckermann A, et al. Efficacy of psychosocial intervention in patients with mild Alzheimer's disease: the multicentre, rater blinded, randomised Danish Alzheimer Intervention Study (DAISY). BMJ 2012;345:e4693.

7. Sørensen LV, Waldorff FB, Waldemar G. Early counselling and support for patients with mild Alzheimer's disease and their caregivers: a qualitative study on outcome. Aging Ment Health 2008;12:444-50.

8. Phung TKT, Waldorff FB, Buss DV, et al. Three-year follow-up on the efficacy of psychosocial interventions for patients with mild dementia and their caregivers: the multicentre, rater blinded, randomized Danish Alzheimer Intervention Study (DAISY). BMJ Open 2013;3:e003584.

9. Mittelman MS, Haley WE, Clay OJ, et al. Improving caregiver well-being delays nursing home placement of patients with Alzheimer disease. Neurology 2006;67:1592-99.

10. Nichols LO, Chang C, Lummus A, et al. The cost-effectiveness of a behavior intervention with caregivers of patients with Alzheimer's disease. J Am Geriatr Soc 2008;56:413-20.

11. Belle SH, Burgio L, Burns R, et al. Enhancing the quality of life of dementia caregivers from different ethnic or racial groups: a randomized, controlled trial. Ann Intern Med 2006;145: 727-38.

12. Callahan $\mathrm{CM}$, Boustani MA, Unverzagt FW, et al. Effectiveness of collaborative care for older adults with Alzheimer disease in primary care: a randomized controlled trial. JAMA 2006;295:2148-57.

13. Søgaard R, Sørensen J, Waldorff FB, et al. Cost analysis of early psychosocial intervention in Alzheimer's disease. Dement Geriatr Cogn Disord 2013;37:141-53.

14. Søgaard R, Sørensen J, Waldorff FB, et al. Private costs almost equal health care costs when intervening in mild Alzheimer's: a cohort study alongside the DAISY trial. BMC Health Serv Res 2009;9:215.

15. Kruse M, Christiansen T. Register-based studies of healthcare costs Scand J Public Health 2011;39(7 Suppl):206-9.

16. Jonsson L, Eriksdotter JM, Kilander L, et al. Determinants of costs of care for patients with Alzheimer's disease. Int J Geriatr Psychiatry 2006;21:449-59.

17. Wimo A, Nordberg G. Validity and reliability of assessments of time. Comparisons of direct observations and estimates of time by the use of the resource utilization in dementia (RUD)-instrument. Arch Gerontol Geriatr 2007;44:71-81.

18. The EuroQol Group. EuroQol-a new facility for the measurement of health-related quality of life. Health Policy 1990;16:199-208.

19. Wittrup-Jensen KU, Lauridsen J, Gudex C, et al. Generation of a Danish TTO value set for EQ-5D health states. Scand J Public Health 2009;37:459-66.

20. Helweg-Larsen K. The Danish Register of causes of death. Scand J Public Health 2011;39(7 Suppl):26-9.

21. Sterne JA, White IR, Carlin JB, et al. Multiple imputation for missing data in epidemiological and clinical research: potential and pitfalls. BMJ 2009;338:b2393.

22. White IR, Royston P, Wood AM. Multiple imputation using chained equations: Issues and guidance for practice. Stat Med 2011;30:377-99.

23. Carpenter J, Bithell J. Bootstrap confidence intervals: when, which, what? A practical guide for medical statisticians. Stat Med 2000;19:1141-64.

24. Manca A, Hawkins N, Sculpher MJ. Estimating mean QALYs in trial-based cost-effectiveness analysis: the importance of controlling for baseline utility. Health Econ 2005;14:487-96.

25. Hoch JS, Briggs AH, Willan AR. Something old, something new, something borrowed, something blue: a framework for the marriage of health econometrics and cost-effectiveness analysis. Health Econ 2002;11:415-30.

26. Stinnett AA, Mullahy J. Net health benefits: a new framework for the analysis of uncertainty in cost-effectiveness analysis. Med Decis Making 1998;18(2 Suppl):S68-80.

27. Koopmanschap MA, van Exel JN, van den Berg B, et al. An overview of methods and applications to value informal care in economic evaluations of healthcare. Pharmacoeconomics 2008;26:269-80.

28. Zhao H, Novella JL, Drame M, et al. Factors associated with caregivers' underestimation of quality of life in patients with Alzheimer's disease. Dement Geriatr Cogn Disord 2012;33:11-17.

29. Bhattacharya S, Vogel A, Hansen ML, et al. Generic and disease-specific measures of quality of life in patients with mild Alzheimer's disease. Dement Geriatr Cogn Disord 2010;30:327-33.

30. Graff MJ, Adang EM, Vernooij-Dassen MJ, et al. Community occupational therapy for older patients with dementia and their care givers: cost effectiveness study. BMJ 2008;336:134-8.

31. Woods RT, Bruce E, Edwards RT, et al. REMCARE: reminiscence groups for people with dementia and their family caregiverseffectiveness and cost-effectiveness pragmatic multicentre randomised trial. Health Technol Assess 2012;16:v-xv, 1-116.

32. Martikainen J, Valtonen H, Pirttila T. Potential cost-effectiveness of a family-based program in mild Alzheimer's disease patients. Eur J Health Econ 2004;5:136-42.

33. Weimer DL, Sager MA. Early identification and treatment of Alzheimer's disease: social and fiscal outcomes. Alzheimers Dement 2009;5:215-26. 